கலைந்த கனவுகள் நாவலில் பஞ்சாலைத் தொழிலாளர்களின் சமூக நிலை பா. நாகேஸ்வரி அ, *

அ தமிழ்த்துறை, ஸ்ரீ எஸ். இராமசாமி நாயுடு ஞாபகார்த்தக் கல்லூரி, சாத்தூர்-626203, தமிழ்நாடு, இந்தியா.

\title{
The Social Status of Panchala Workers in the Novel Dreams
}

\section{P. Nageswari a, ${ }^{*}$}

a Department of Tamil, Sri S. Ramasamy Naidu Memorial College, Sattur-626203, Tamil Nadu, India.

\section{* Corresponding Author: nagesarumugathai@gmail.com}

Received: 23-03-2021

Revised: 20-01-2022

Accepted: 28-01-2022

Published: 25-02-2022

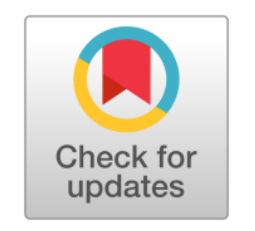

\begin{abstract}
The novel 'Dispersed Dreams' is a description of the social position of textile workers. 'KalaiKada Kanavukkal' by R. Vichalan has been described in the social situation of the people who go to the textile symposium in coimbatore, Tiruppur, Dindigul and Erode districts. The novel also shows the large number of children in the society who go to the textile strip and the cruel tyranny of women in the name of the scheme. Society is a group of people living as a group. The social status of the community will change according to the change in the environment. Illustrates the life of the working community in the panchayats. Lack of rainfall causes agricultural damage. The middlemen are taking advantage of the poverty of the people. People join the plant to alleviate their poverty. Lets learn about the plight of working women staying in textile and their place of residence. The exploitation of workers' labor can also be seen. The novel 'Dispersed Dreams' highlights the problem of the life of textile workers.
\end{abstract}

Keywords: Cotton, Community, Intermediary, Scheme.

\section{முன்னுரை}

சமுதாயம் என்பது மிகப்பெரிய மக்கள் குழு. அது தனக்கென்று ஒரு குறிப்பிட்ட பண்பாட்டைக் கொண்டது. அவ்வாறாக இருக்கும் சமுதாயத்தில் ஒரு பகுதியாக விளங்கும் பஞ்சாலைகளில் வேலை செய்யும் மக்களின் சமுதாயத்தை எடுத்துக்காட்டுவதாக ‘கலைந்த கனவுகள்’ நாவல் உள்ளது. இந்நாவலில் கோவை, திருப்பூர், திண்டுக்கல், ஈரோடு போன்ற மாவட்டங்களில் வாழும் மக்கள் வேளாண்மைத் தொழிலை முதன்மைத் தொழிலாகக் கொண்டு வாழ்கின்றனர். அத்தொழில் பாதிக்கப்படும் நிலையில் பஞ்சாலைக்குச் செல்கின்றனர். அதுவே அம்மக்களின் தொழிலாக மாறுகிறது. பஞ்சாலைக்குச் செல்லும் மக்களின் வாழ்வியல் சமுதாயத்தை எடுத்துக்காட்டும் விதமாக இக்கட்டுரை அமைகிறது.

\section{வேளாண்மை பாதிப்பு நிலை}

மக்களின் வாழ்வியலுக்கு ஏற்ற தொழில்களில் முதன்மையானதாக எண்ணப்படுவது ‘வேளாண்மை’ ஆகும். இத்தொழில் 'இந்தியாவின் முதுகெலும்பு’ என்ற பெருமையைப் பெற்றுள்ளது. வேளாண்மைச் சிறப்பை சங்ககாலம் தொட்டு இன்றுவரையிலும் அவற்றை உணரமுடிகிறது. சங்ககால மக்கள் தாங்கள் வாழும் நிலத்திற்கு ஏற்ற தொழிலினைச் செய்து வாழ்ந்தனர். அவற்றில் மருதநில மக்கள் 
வேளாண்மைத் தொழிலில் ஈடுபட்டும் தங்களுக்குத் தேவைானப் பொருட்களைப் பண்டமாற்று முறையில் பெற்று வாழ்ந்து வந்தனர். நீதிஇலக்கியக் காலத்திலும் உழவின் சிறப்பை வள்ளுவர்,

“சுழன்றும்ஏர்ப் பின்னது உலகம் அதனால்

உழந்தும் உழவே தலை"

(Thiruvalluvar, 2015)

எனக் குறிப்பிடுகிறார். உலகமானது பல தொழில்களின் பின்னால் சுழன்றாலும் அது ஏர்த்தொழிலை - உழவுத்தொழிலைச் சார்ந்தே நிற்க வேண்டும். ‘உழவே தலைசிறந்த தொழில்' என்பதை இக்குறள் விளக்குகின்றது. உழவின் சிறப்பை வள்ளுவர் இங்குக் குறிப்பிட்டுள்ளார். இன்றைய காலத்தில் வேளாண்மையானது அதிக அளவில் பாதிப்புக்கு உள்ளாகியுள்ளது. காலமாற்றத்தின் காரணத்தாலும் மழை பெய்ய வேண்டிய காலத்தில் பெய்யாமல் இருப்பதாலும் நிலங்கள் பிளவுப்பட்டு எங்கும் வறட்சியோடு இருக்கும் சூழலே இன்று காணப்படுகிறது. இன்றைய வேளாண்மைச் சூழலைப் பிரதிபலித்துக் காட்டும் விதமாக ‘கலைந்த கனவுகள்’ நாவலில் மழை இரண்டு வருடங்கள் பெய்யாமல் இருந்ததால் நிலங்கள் வறட்சியுற்றுக் காணப்படும் நிலையை புலப்படுத்துகின்றன. இவ்வாறு வறட்சி ஏற்பட்ட நிலத்தைக் கண்ட விவசாயியின் மனவேதனையை ஆசிரியர் எடுத்துக்காட்டுகிறார். மழை இல்லாத காரணத்தினால் நிலங்கள் வறட்சியோடும் பசுமை இல்லாமலும் காணப்படுகின்றன. வேளாண்மைத் தொழிலானது இன்றைய அறிவியல் வளர்ச்சியடைந்த காலத்தில் எளிமையான முறையில் செய்ய ஏதுவாக இருந்தாலும் மழை இல்லாத காலத்தில் விவசாய நிலமானது நீர்ச்சத்து இழந்து காணப்படுவதால் விவசாயம் இல்லாமல் போகிறது என்பதையும் வறட்சி காரணமாக விலங்குகளுக்கும் உணவு அற்ற நிலை இருப்பதையும் இந்நாவலில் காணமுடிகிறது. மக்களின் வாழ்க்கைக்கு மழை மிகவும் முக்கியமானது, அது பெய்ய வேண்டிய காலக்கட்டத்தில் பெய்யாமல் போனால் இவ்வாறே வறட்சியுற்று நிலங்களும், விலங்குகளும் தண்ணீர் இன்றி காணப்படும்.

\section{இடைத்தரகர் சூழல்}

இடைத்தரகர் என்பவர் தெரிந்த நபரை தெரியாதவருக்கு அறிமுகம் செய்து வைப்பவர் ஆவார். இந்நாவலானது இடைத்தரகர் சூழலை மையமிட்டதாக அமைகிறது. இடைத்தரகர்கள் வேளாண்மையால் பாதிக்கப்பட்ட குடும்பம், பெண்களுக்குத் திருமணம் செய்து கொடுக்க முடியாத வறுமையில் வாடும் குடும்பம் போன்ற குடும்பங்களின் சூழ்நிலையை அறிந்து கொண்டு, அவர்களைப் பஞ்சாலைகளில் சேர்த்து விட்டு, அவற்றின் மூலம் கமிஷன் பெறுகின்றனர் என்று இடைத்தரகர் பற்றிய செய்திகளை 'கலைந்த கனவுகள்’ நாவல் எடுத்துரைக்கின்றது.

இடைத்தரகர்கள் அவர்களின் குடும்பச்சூழல்களை அறிந்து கொண்டு அவற்றைத் தங்களுக்குச் சாதகமாகப் பயன்படுத்திக் கொள்கின்றனர். அவற்றைப் பெறுவதற்காகப் பொய் கூறி வேலை பார்த்துக் கொண்டே பிள்ளைகளுக்கு கல்வி கிடைக்கும், கல்யாணத்துக்கு வேண்டிய காசைத் தாங்களே சம்பாதித்துக் கொள்வார்கள் போன்ற பொய்யான வாக்குறுதிகளைக் கொடுத்து அவர்களைச் சேர்த்து விடுகின்றனர். இவ்வாறான தேொழுகும் பேச்சைக் கேட்டு ஏமாந்து மக்கள் தங்கள் பிள்ளைகளை ஆலையில் சேர்த்து விடுகின்றனர். அவர்களின் பேச்சால் தன்னுடைய குழந்தைக்கு நல்லது நடக்கும் என்ற எண்ணத்திலும், குடும்பச் சூழல் காரணமாகவும் வேலைக்கு அனுப்புகின்றனர். இப்படியாக, இடைத்தரகர்களின் நிலையானது அமைந்துள்ளது என்பதை ‘கலைந்த கனவுகள்’ நாவலானது காட்டுகின்றது.

\section{தொழில்}

தொழில் தான் வாழும் சமுதாயத்தின் சூழ்நிலைக்கு ஏற்ப அமையும். சங்க இலக்கியத்தில் அவ்வவ் நிலங்களுக்கு ஏற்ப தொழில் செய்து வாழ்ந்தனர் என்று கூறப்படுகிறது. இதனைக் காட்டும் விதமாக திருக்குறளில்,

"பிறப்பொக்கும் எல்லா உயிர்க்கும் சிறப்பொவ்வா 
செய்தொழில் வேற்றுமை யான்"

(Thiruvalluvar, 2015)

என வழங்கப்படுகிறது. பிறப்பால் அனைவரும் சமமாகக் கருதப்பட்டாலும் அவரவர் தொழிலைக் கொண்டே வேறுபாடு அறிய முடியும் என்பதை இக்குறள் விளக்குகிறது. ஒவ்வொரு காலத்திலும் மக்கள் சமுதாய நிலைக்கு ஏற்பத் தொழில் அமையும். அவ்வாறாகத் தோன்றிய முதலாளித்துவ சமுதாயக் காலத்தில் தான் சுயலாபத்திற்காக தொழில் என்ற நிலை ஏற்பட்டது. இந்நாவலில் வேளாண்மைத் தொழில் பாதிக்கப்பட்ட நிலையில் மில் ஆலைக்கு சென்று வேலைப் பார்ப்பதாக காட்டப்படுகிறது.

மழையின்மையால் கடும் வறட்சி ஏற்பட்ட சூழலில் பஞ்சாலை நிறுவப்படுகிறது. தங்களின் குடும்ப வறுமையைப் போக்கும் விதமாக உள்ளதால் அவற்றில் வேலைக்குச் சேர்கின்றனர். அதனைப் பயன்படுத்திக் கொண்டு குழந்தைகளையும், இளம்பெண்களை கொத்தடிமை முறைகளிலும் வேலை வாங்கப்படுகின்றனர். இரண்டு வகையான முறைகளில் வேலை வாங்கப்படுகிறது. அவை:

1. குழந்தைத் தொழிலாளர் முறை

2. இளம்பெண்கள் கொத்தடிமை முறை ஆகியவையாகும்.

\section{1.குழந்தைத் தொழிலாளர் முறை}

குழந்தைகள் தொழிற்சாலைகளில் வேலையில் ஈடுபடுத்தப்படுவது தொழிற்புரட்சிக் காலத்தில் தான் தொடங்கியது. தொழிற்புரட்சியின் காரணமாகத் தொழிற்சாலைகள் மிகுந்த அளவில் தொடங்கப்பட்டதும் அவற்றுக்கு அளவற்ற தொழிலாளர்கள் தேவைப்பட்டனர். அந்தத் தேவையைச் சமாளிக்கக் குழந்தைகளும் தொழிலாளர்களாக வேலையில் அமர்த்தப்பட்டனர். இதனை

“குறைந்த ஊதியத்தில் குழந்தைகள் வேலைக்குக் கிடைத்தால் தொழிலதிபர்கள் மிகுதியான குழந்தைகளைத் தொழிற்சாலைகளில் வேலைக்கு எடுத்துக் கொண்டனர்." (Balusami, 2004)

என்று விளக்குகின்றது. தொழில் நிறுவனங்கள் குழந்தைகளை மையமாகக் கொண்டு நடைபெறுகின்றன. குழந்தைகள் எவ்வளவு கடுமையான வேலையாக இருந்தாலும் செய்வர். வருமானத்திற்காக அவர்கள் சொல்லும் வேலையைத் தட்டாமல் செய்வர் என்ற எண்ணம் முதாலித்துவ வர்க்கத்தால் உருவாக்கப்பட்டது. ‘குழந்தைத் தொழிலாளர் இருத்தல் கூடாது’ என்று சட்டம் குறிப்பிடுகிறது. இந்திய அரசியலமைப்பும் அரசின் சட்டங்களும் பதினான்கு வயதுக்குக் குறைந்தோர் வேலைக்கு அமர்த்தப்படுவதைக் கண்டிக்கிறது. ஆனால், பஞ்சாலைவர்க்கம் குழந்தைகளைக் குறிவைத்து அதிகமான வேலை வாங்கிக் குறைவான சம்பளம் வழங்குகிறது. குழந்தைகளைத் தொழிலுக்கு உட்படுத்துகின்றனர் என்பதை இந்நாவலில் அறியமுடிகிறது.

குழந்தைகள் தன் குடும்ப வறுமையின் காரணமாகவும் தந்தை குடிகாரராக இருப்பதாலும் வேலைக்குச் செல்கின்றனர் என ஆசிரியர் எடுத்துக்காட்டுகின்றார். ‘ராணி’ என்ற பெண்ணும் வறுமையின் காரணமாக அவ்வாலையில் இடைத்தரகரின் மூலமாகச் சேர்க்கப்படுகிறாள். குழந்தைகளை தொழிலுக்கு உட்படுத்தக்கூடாது என்று அறிந்தும் அவர்களை வேலைக்கு அமர்த்தி அதிகமான வேலைப்பளுவைக் கொடுக்கின்றனர்.

இவ்வாறாக, குடும்ப வறுமையைப் பயன்படுத்திக் கொண்டு அவர்களை வேலையில் சேர்த்துவிடுகின்றனர். அக்குழந்தைகள் இன்புற்று விளையாட வேண்டியவர்கள், சோர்வுடன் உடம்பில் நோய் ஏற்பட்டுக் காணப்படுகின்றனர். அதிகமான வேலைப்பளுவைக் கொடுத்து அவற்றைத் தாங்கமுடியாமல் ராணிக்கு ‘எலும்புருக்கி நோய்’ ஏற்படுகிறது. தன் குடும்ப வறுமைக் காரணமாகவும், தனக்கு ேேண்டியத் தேவைகளை தானே பூர்த்தி செய்துக் கொள்ளமுடியும் என்ற காரணத்திற்காக குழந்தைகள் வேலைக்குச் ெல்கின்றனர். தொழிலின் காரணமாக ஒருவர் அடிமைப்படுத்தப்படுகின்ற நிலையை இன்றைய காலகட்டத்திலும் காணமுடிகிறது. 
2. இளம்பெண்கள் கொத்தடிமை முறை

பஞ்சாலைகளில் கொத்தடிமை முறைகளிலு வாங்கப்பட்டது. "அடிமைப்படுத்தப்படுகின்ற மனிதன் பிணைத் தொழிலாளி அல்லது கொத்தடிமை எனச் சொல்லப்படுகிறான்" (Balusami, 2004).

என்று குறிப்பிடுகிறது. நாவலில் ஸ்கீம் என்ற திட்டத்தில் திருமணத்துக்கு வேண்டிய செலவுக்கான மொத்தத் தொகையை “சுமங்கலித் திட்டம், மாங்கல்யத் திட்டம்” போன்ற பெயர்களில் கொத்தடிமை முறையில் வேலை வாங்கப்படுகிறது.

அடிமை முறையில் ஸ்கீம் என்ற திட்டத்தின் பெயரில் மூன்று, நான்கு வருடம் தொடர்ந்து வேலை வாங்குகின்றனர். மேலும் அவற்றின் மூலமாக வேலைக்கு நியமிக்கப்பட்டு சரியான உணவு இல்லாமலும், கழிவறைக்குச் சென்றால் அங்கே ஓய்வெடுப்பார்கள் என்று எண்ணி அங்குச் சுத்தம் இல்லாமலும், அவசர வேலைக் காலத்தில் மில்லில் தங்கி வேலை செய்பவர்களை வேண்டிய நேரத்தில் வேலை வாங்குவது போன்ற ‘கொத்தடிமை முறை’ காணப்படுகிறது. அதிகமாக வேலை வாங்குதல், சிறிது நேரம் ஓய்வு என்ற நிலை இல்லாது தொடர்ந்து வேலை வாங்குதல் போன்ற கொடுமையான நிலையில் வேலை செய்தனர். இவ்வாறு தொடர்ந்து வேலை பார்ப்பதால் நோய் ஏற்படுகிறது. இத்தகைய முறைகளில் வேலை வாங்கப்பட்டச் சூழலை ‘கலைந்த கனவுகள்’ நாவல் காட்டுகின்றது.

\section{தங்கும் இடத்தின் கொடுமை நிலை}

தொழிலாளர்களின் தங்கும் இடமானது பாதுகாப்பாகவும் நலம் பயப்பதாகவும் அமைதல் வேண்டும். ஆனால் சில இடங்களில் தூய்மையற்று, அவற்றால் நோய் ஏற்படுவதற்குக் காரணமாகவும் அவை திகழ்கின்றன. 'கலைந்த கனவுகள்' நாவலில், பஞ்சாலைகளில் தங்கி வேலை செய்யும் பெண்கள் பல்வேறான இன்னல்களை அனுபவிக்கின்றனர்.

பஞ்சாலைகளில் வேலை செய்பவர்களின் அறைகள் சிறியதாகவும் அவற்றிலே தங்கும் நபர்களின் எண்ணிக்கை அதிகமாகவும் காணப்படுவதை நாவல் மூலம் அறியமுடிகிறது. அந்த அறையானது குப்பைக் கூழமாகவும் துர்நாற்றம் வீசுவதாகவும் காணப்படுகிறது. அதிகமான வேலை வாங்கும் விதமாக அறைகளில் தங்குபவர்களுக்கு ஒவ்வொரு நேரத்தில் வேலை என்ற சிப்ட் முறையில் வேலை பார்ப்பதாகக் காட்டப்படுகிறது. மேலும் அங்குள்ள கழிவறைகள் தூய்மையற்ற நிலையிலும் அவற்றால் நோய் ஏற்படுவதும் காட்டப்படுகிறது. குளியலறை, கழிவறை சுத்தமற்ற நிலையிலும் அவை சுத்தமாக இருந்தால் அங்கே சென்று ஓய்வெடுப்பார்கள் என்றும் கருதி அவ்வாறாக வைத்துள்ளனர். இவ்வாறாக 'தங்கும் இடத்தின் நிலை’ காணப்படுகிறது.

\section{அதிகாரிகளின் கொடுமை முறை}

நிறுவனங்களில் வேலை செய்பவர்கள் முதலாளிகள் கூறும் செயல்களுக்கு அடிமைப்பட்டு வாழ்கின்றனர். இதனை,

“முதலாளித்துவ சமுதாயம் என்பது முதலாளிகளால் மட்டும் ஆக்கப்பட்ட சமுதாயமன்று. அது முதலாளிகளை ஒரு - புறமும் ஏழைகளை ஒரு - புறமும் பிரித்து அந்நிலையிலும் அவர்களை நீடித்து நிற்கவிடாமல் ஓயாது சுழற்றி ஆட்டி வரும் முறை ஆகும். அதனை ஆக்கியவர்கள் முதலாளிகள், தொழிலாளிகள், நடுத்தர வகுப்பினர்ஆகிய எல்லா மக்களுமே” என்று கு. விவேகானந்தம் விளக்குகிறார் (Vivekanandan, 2012).

நாவலில் நிறுவன அதிகாரிகள் தங்களிடம் வேலை செய்பவர்களிடம் அதிகமான வேலை வாங்கியும் அவற்றிற்கேற்ற ஊதியம் குறைவாகக் கொடுத்தும், வேலை செய்பவர்களின் இறப்புக்கு நிறுவனம் காரணம் இல்லை என்ற பொய்யான வாக்குமூலம் கொடுப்பதாகக் காட்டப்படுகிறது. நாவலில் முதலாளித்துவ நிலை முதலாளிகள் தங்கள் சுயநலத்திற்காக சாதியமுறையைப் பயன்படுத்துகின்றனர் என்பதனை நாவல் எடுத்துரைக்கின்றன. 
முதலாளிகள் தங்கள் வாழ்வின் முன்னேற்றத்திற்காக ‘சாதி’ என்ற பெயரில் ஆட்படுத்தப்பட்டு, அவர்களை அடிமைப்படுத்தி வேலையில் அமர்த்தி, அவர்களுக்குத் தேவையானவற்றை நிறைவேற்றிக் கொள்கின்றனர். இவ்வாறாக முதலாளிகள் மூலதன வலிமையால் உழைப்பைச் சுரண்டி இன்னொரு ஆலையின் உரிமையாளராகி விடுகின்றனர். ஆனால், முன்னேற்றத்திற்குக் காரணமாக இருக்கின்ற தொழிலாளர்களை மறந்து விடுகின்றனர். மேலும் அதிகமான வேலையும் வாங்குகின்றனர் என்பதையும் அறியமுடிகிறது.

\section{உழைப்புச் சுரண்டல்}

சுரண்டல் என்பது ஒரு மனிதனின் உழைப்பு, ஆற்றல் ஆகியவற்றை தன்னுடைய இலாபத்திற்காகச் சுரண்டுவது ஆகும். அதாவது,

“உற்பத்தி முறையாலும் அங்காடி முறையாலும் ஒருவருக்குச் சேர வேண்டிய தொகையை மற்றவர் எடுத்துக் கொள்ளுதல் சுரண்டல் எனப்படும்" (Balusami, 2004).

விளக்குகிறது. சுரண்டலின் மூலமாக ஒருவரின் உழைப்பு, ஆற்றலை எடுத்துக் கொள்கிறது. உழைப்புச் சுரண்டலானது ஒருவனது உழைப்பை மட்டும் கடுமையாக வாங்கிவிட்டு அதற்குரிய கூலியைக் கொடுக்காமல் ஏமாற்றுவது உழைப்புச் சுரண்டலாகும். இவற்றையே, மேலும்

“சுரண்டல் என்பது ஒருவருக்குரியதை மற்றவர், உரியவருக்குத் தெரியாமல் அவர் உணராமல் சிறுகச் சிறுக எடுத்துக் கொள்வதென்றும் கூறலாம். இது கொசு, மூட்டைப்பூச்சி ஆகியவை நாம் தூங்கும்போது நமக்குத் தெரியாமல் நமது இரத்தத்தை உறிஞ்சுவதைப் போன்றதாகும்" (Kurusami, 1985).

என பகர்கின்றது. இவற்றைப் போன்றே இந்நாவலில் மக்களின் உழைப்பைச் சுரண்டி அவற்றிற்கேற்ற ஊதியம் கொடுக்காமல் ஏமாற்றுவதைக் காணமுடிகிறது.

அதிகமான உழைப்பை வாங்கிக் கொண்டு குறைவான சம்பளத்தை கொடுக்கின்றனர். மேலும் ஓய்வு எடுக்கக் கூடாது என்ற நிலையில் அதிகமான வேலையை வாங்குகின்றனர். உழைப்புச்சுரண்டல் மூலமாக அவர்களின் ஆற்றலை உறிஞ்சி நோய் வருவதற்கு காரணமாக அமைகின்றனர். முதலாளிகள் தாங்கள் முன்னேறுவதற்காக தொழிலாளர்களின் வேலைப் பளுவை அதிகமாக்கி உழைப்பைச் சுரண்டுவது இங்குக் காணப்படுகிறது.

\section{மனிதநேயமின்மை}

உலகில் மனிதன் தோன்றிய காலத்தில் இருந்து ஒரு உயிர் பிற உயிரைக் கண்டு இரக்கம் கொள்வதே மனிதநேயம் ஆகும். சங்க இலக்கியங்களிலும் பிற இலக்கியங்களிலும் மனிதநேயம் பற்றியக் குறிப்பு காணப்படுகிறது. அவற்றில் அரசனின் மனிதநேயப் பண்பானது

மரம்சா மருந்துங் கொள்ளார் மாந்தர்

உரம்சாச் செய்யார் உயர்தவம் வளம்கெடப்

பொன்னும் கொள்ளார் மன்னர்"

(Seyapal, Ira, 2004)

என்ற பாடல் வரிகளின் மூலம் அறியமுடிகிறது. அரசன் வருவாய் ஒன்றையே நோக்கமாகக் கொள்ளக் கூடாது. அவ்வாறிருப்பின் அது மனித உரிமையை மீறும் செயலாகும். மருத்துவக் குணம் மிக்க ஒரு மரத்தை மக்கள் அம்மரம் சாகும்படி எடுக்கமாட்டார்கள். அதுபோல, அரசனும் மக்கள் வளம் கெடும்படி வரி வாங்கக்கூடாது என்ற கருத்தை விளக்குகிறது. மணிமேகலையிலும் மனிதநேயம் குறித்து,

“காணா உயிர்க்கும் கையற்று ஏங்கி

உண்ணா நோன்பொடு உயவல் யானையின்" மணிமேகலை, (Puliyur Kasiken,1961)

சமணத்துறவிகள் எவ்வுயிர்க்கும் துன்பம் செய்யாதவர்கள் என்ற செய்தியைக் கூறப்பட்டுள்ளது. இவ்வாறாக மனிதநேயம் ம்க்கவராக இருத்தல் வேண்டும் என்று இலக்கியங்கள் குறிப்பிடுகின்றது. 
மனிதநேய மற்ற நிலையும் காணப்படுகிறது. இன்றைய காலத்தில் மனிதநேயம் என்பது முற்றிலும் அழிந்துவிட்டத் தன்மை காணப்படுகிறது. ஒருவர் விபத்துக்குட்பட்டு சாலையில் கிடக்கும் பொழுது உதவாமல் அவற்றை புகைப்படம் எடுத்து பிறருக்கு அனுப்புகின்ற நிலை இன்று நிலவுகிறது. நாவலிலும் ஆலையில் வேலைப்பார்ப்பவர்கள் வேலைப்பளுவின் காரணமாக நோய் ஏற்படுகின்றன அதற்காக நஷ்ட ஈடு கேட்டு செல்லுகையில் அவற்றை மதிக்காமல் வேலை செய்பவர்கள் மீதே குற்றத்தைச் சுமத்துகின்றனர்.

வேலைக் காரணமாக விரலை இழந்ததற்கு நஷ்ட ஈடு கேட்டும் அவை நாங்கள் மருத்துவத்திற்கு செலவளித்ததற்கும், நீங்கள் வாங்கிய முன்பணத்திற்கும் சரியாகிவிட்டது என்பது போல் கூறி அவர்களுக்கு எதுவும் கிடைக்காமல் செய்தனர். காலம் முழுதும் தன் ஒரே கையை வைத்துக் கொண்டு அவற்றால் அவளுக்கு வரும் திருமணம் தட்டிக் கழித்துக் கொண்டே போனது இறுதியில் பைத்தியமாக மாறிவிட்டால். இதனைப் போன்று ம்ல்லில் வேலைப்பார்த்த ராணிக்கும் வேலைபளுவினால் டி. பி நோய் ஏற்பட்டது அதை நிர்வாகம் மறைத்து அவளுக்கு எயட்ஸ் நோய் இருப்பதாகக் கூறி அவளது இறப்புக்கு அதுவே காரணமாயிற்று என்று கூறி உண்மையை மறைத்தனர் அவளது குணத்தையை தவறாகக் கூறும் நிலையை காணமுடிகிறது. இவ்வாறான சூழல்கள் இன்றைய காலத்தில் எளிதாகிவிட்டன. பணம் படைத்தவர்கள் அவர்களின் தேவைக்கேற்ப தொழிலாளியைப் பயன்படுத்தும் நிலை காணப்படுகிறது. இவ்வாறான 'மனிதநேயமற்ற தன்மையே’ இன்று காணப்படுகிறது.

\section{நோய்}

நோய் என்ற சொல்லினைப் 'பிணி’ என்றும் அழைத்தனர். இச்சொற்கள் துன்பத்தைத் தருவது என்ற பொருளில் வழங்கப்பட்டது. நோயின்றி வாழ்வதே சிறந்த வாழ்வாகக் கருதப்படுகிறது. இதனை, “நோயற்ற வாழ்வே குறைவற்ற செல்வம்” என்ற பழமொழியின் மூலம் உணரமுடியும். மனிதன் உடலோ, மனமோ அல்லது இவ்விரண்டுமோ நோய்வாய்ப்பட்டிருந்தால் மனிதன் அன்றாடம் தாம் செய்யும் செயல்களை செய்ய முடியாது. இளமையானாலும் முதுமையானாலும் மனிதன் நோயின்றி வாழ்வதே சிறந்ததாகும். நாவலில் பஞ்சாலைகளில் அதிகமான வேலைப்பளுவின் காரணமாக இளம்வயதில் நோய்வாய்ப்பட்டுத் துன்பப்படுகின்றனர்.

அதிகமான வேலையாலும் தூசியின் காரணமாகவும் ‘நோய் ஏற்படுகின்றது. ஆலையில் அதிகமான வேலை வாங்கப்பட்டு கொடுமையான நோய் வருவதற்கும் காரணமாக உள்ளன. அந்நோயினால் தன் வேலையைக் கூட தன்னால் செய்து கொள்ள முடியாமல் துன்பப்படுகின்றனர். நோய் காரணமாக ஒரு பெண் உடல்மெலிந்து சோளக்காட்டு பொம்மையைப் போல் காட்சியளிக்கின்றாள் அதனைக்கண்டு வேதனையடைவதாகக் காட்டப்படுகிறது. நோயினால் தனது உடல்நிலை பாதிக்கப்பட்டு உருவஅழகு இன்றி காணப்படுகின்றனர். இவ்வாறாக, நோயினால் பாதிக்கப்பட்டவரை இந்நாவலில் காணமுடிகிறது. நோய் இல்லாமல் இருப்பதே சிறந்த வாழ்வாகும். நோயானது ஒருவரைத் தாக்கினால் அவரது மனம் அதை விட்டு விலகாமல் தன்னை ஒரு சோம்பல் தன்மையுடன் காட்டும். நோயின்றி இருப்பதே இன்பமான வாழ்விற்கு வழிவகுக்கும்.

\section{முடிவுரை}

சமுதாயம் என்பது குழுவாக வாழும் மக்கள் தொகுதி ஆகும். அச்சமுதாயத்தில் நிகழும் சூழல்மாற்றத்திற்கேற்ப சமுதாய நிலையும் மாற்றம் பெற்றுக் காணப்படும். பஞ்சாலைகளில் வேலை செய்யும் சமுகத்தினரின் வாழ்வை எடுத்துக்காட்டுகிறது. மழையின்மையால் வேளாண்மை பாதிப்பு ஏற்படுகிறது. இடைத்தரகர் மக்களின் வறுமையைப் பயன்படுத்திக் கொள்கின்றனர். மக்கள் தங்கள் வறுமையைப் போக்குவதற்காக ஆலையில் சேர்கின்றனர். பஞ்சாலைகளில் தங்கி வேலை செய்யும் பெண்களின் கொடுமை நிலையினையும் அவர்கள் தங்கும் இடத்தினையும் பற்றி தெரிந்து கொள்ள முடிகிறது. தொழிலாளர்களின் உழைப்பைச் சுரண்டுவதையும் காணமுடிகிறது.

இவ்வாறு கலைந்த கனவுகள் நாவலில் திண்டுக்கல்லில் உள்ள பஞ்சாலைகளில் வேலை செய்யும் மக்களின் நிலையையும் அங்கு ஏற்பட்ட கொடுமைகளை எடுத்துக் கூறுவதாக இந்நாவல் அமைகிறது. 
இவற்றுக்குத் தீர்வாக இறுதியில் கொடுமையை எதிர்த்துப் பெண்கள் அனைவரும் போராட்டம் செய்கின்றனர் என்பது இங்கு தெளிவாகிறது. இவற்றின் மூலமாக பஞ்சாலைகளில் தொழில் செய்யும் மக்களின் சமூக வாழ்க்கை நிலையானது அமைந்துள்ள தன்மையை எடுத்துக் காட்டுவதாக இக்கட்டுரை அமைகிறது.

\section{References}

Balusami, Na., (2004) Valviyal kalanjiyam Volume $(7,8,9)$, Tamil University, Thanjavur, Tamil Nadu, India.

Kurusami, MP., (1985) Economics of Exploitation, Christian Literary Society, Chennai, Tamil Nadu, India.

Puliyur Kasiken, (1961) Manimegalai, Parry Station, Chennai, Tamil Nadu, India.

Seyapal, Ira., (2004) Natrinai, New Century Book House Pvt Ltd Chennai, Tamil Nadu, India.

Thiruvalluvar, (2015) Thirukkural, Aruna Publishing, Chennai, Tamil Nadu, India.

Vivekanandan, K., (2012) Annayiyam, Padma Publications, Chennai, India.

Funding: No funding was received for conducting this study.

Conflict of Interest: The Author has no conflicts of interest to declare that they are relevant to the content of this article.

\section{About the License:}

\section{(ㄷ)(1)}

Attribution 4.0 International (CC BY 4.0)
(C) The Author 2022. The text of this article is licensed under a Creative Commons Attribution 4.0 International License 